Journal of Medical and Biomedical Sciences (2013) 2(3): 8-16

C) UDS Publishers Limited All Right Reserved 2026-6294

ORIGINAL ARTICLE

doi: http://dx.doi.org/10.4314/jmbs.v2i3.2

\title{
Links among motivation, socio-demographic characteristics and low physical activity level among a group of Nigerian patients with type 2 diabetes
}

\author{
A.F. Adeniyi1 ${ }^{1}$, O.O. Ogwumike ${ }^{1}$, C.G. John-Chu ${ }^{1}$, A.A. Fasanmade ${ }^{2}$ and J.O. Adeleye ${ }^{2}$ \\ ${ }^{1}$ Department of Physiotherapy, ${ }^{2}$ Department of Medicine, College of Medicine, University of Ibadan, Ibadan, Oyo State, Nigeria
}

\begin{abstract}
While many studies blame low physical activity (PA) among patients with type 2 diabetes mellitus (T2DM) on lack of motivation, others have inconsistently linked it with socio-demographic characteristics. This study investigated the associations among motivation, socio-demographic characteristics and low level participation in physical activity among a group of Nigerian patients with T2DM. A consenting sample of 326 T2DM patients attending diabetes clinics of the two largest hospitals in Ibadan participated in this cross-sectional study. Motivation types and PA levels were assessed using the Motivation for Physical Activity Questionnaire and International Physical Activity Questionnaire respectively. Socio-demographic data were also documented. Regression analysis was used to determine associations at $\mathrm{p}<0.05$. A total of $245(75.2 \%)$ of the participants were intrinsically motivated towards PA but only 108 (33.1\%) were active at moderate-to-high levels. Motivation and duration of diagnosis were not linked with low PA while being female $(\mathrm{OR}=1.57$; $95 \% \mathrm{CI}$ $=1.22-4.19)$, age $>60(\mathrm{OR}=2.42 ; 95 \% \mathrm{CI}=1.91-3.77)$, no education $(\mathrm{OR}=2.21 ; 95 \% \mathrm{CI}=1.64-4.33)$, retirement $(\mathrm{OR}=3.89 ; 95 \% \mathrm{CI}=2.72-6.35)$ and being married $(\mathrm{OR}=1.63 ; 95 \% \mathrm{CI}=1.14-3.66)$ conferred increased risks of low PA. The Nigerian T2DM patients were appropriately motivated but PA was below recommended levels. Low PA was significantly linked with more of the sociodemographic characteristics than motivational factors.
\end{abstract}

Journal of Medical and Biomedical Sciences (2013) 2(3), 8-16

Keywords: Type 2 diabetes; Physical activity; Motivation; Psychological factors; Physical exercise

\section{INTRODUCTION}

A large number of individuals who present with chronic illnesses find it difficult to commence most of the healthcare habits that have been found to be beneficial to their overall health and when they are able to commence the activities, most of them find it difficult to maintain such habits (Amico, 2011). This assertion is also supported by Dunbar-Jacobe and Mortimer-Stephen (2011) who claimed that almost two thirds of persons with chronic illnesses do not follow their treatment prescriptions. One of the prescriptions in the line of management of these patients as shown by systematic reviews is the adoption of

Correspondence: Dr. A.F. Adeniyi, Department of Physiotherapy, College of Medicine, University of Ibadan, Ibadan, Nigeria, E-Mail: adenivifatai@yahoo.co.uk, fadeniyi@,comui.edu.ng regular physical activity at moderate intensities and this has been shown to reduce the risk of having type 2 diabetes mellitus (T2DM) (Jeon et al., 2007).

Physical activity of moderate intensity for at least 150 minutes in a week or at least 90 minutes/week of vigorous aerobic exercise is recommended to enhance cardio-metabolic health of patients with T2DM (Sigal et al., 2006). In spite of the benefits and the laid down prescriptions, most patients with diabetes are inconsistent with their physical activities (Mori et al., 2011). Research findings have also indicated that physical activity is the least maintained of all the self-care efforts (Shigaki et al., 2010). There appears to be a myriad of factors militating against the participation of patients with T2DM in regular physical activity and exercises. Some of the factors include lack of social support (Korkiakangas et al., 2009; Adeniyi et al., 2012), lack 
Motivation/physical activity in diabetes

Adeniyi et al.,

of knowledge of the types of exercise to perform, health problems, pain/difficulty taking part in exercise, lack of local exercise facilities, aversion to exercising in poor weather (Korkiakangas et al., 2009) and a host of other socio-demographic factors (Barrett et al., 2007; Morrato et al., 2007; Huebschmann et al., 2011). However, evidence has also consistently shown that motivation is an important factor in adhering to physical exercises among both healthy and chronically ill populations (Ryan and Deci, 2000; Trost et al., 2004; Biddle and Nigg, 2000; Plotnikoff et al., 2010; Shigaki et al., 2010). This present study therefore presents the physical activity levels of a group of Nigerian patients with T2DM and describes how low physical activity level is associated with the socio-demographic characteristics of the patients and the type of motivation that is being exhibited by the patients.

\section{MATERIALS AND METHODS}

\section{Study sites}

Participants in this cross-sectional study were consenting patients with type 2 diabetes attending the diabetes clinics of the University College Hospital and Ring Road State Hospital, both in Ibadan, Nigeria. The University College Hospital, owned by the Federal Government of Nigeria is a tertiary health care provider and it is the teaching hospital for the training of medical and allied health students. It is also a postgraduate professional medical training centre for resident doctors and other healthcare professionals. The Ring Road State Hospital, owned by the Oyo State Government is a secondary health care provider and it also serves as a source of referral to the University College Hospital. Both hospitals run dedicated diabetes clinics on specific days of the week.

\section{Participants}

The patients were selected based on pre-set criteria. The criteria included being in the age range of 15 to 69 years, having been diagnosed of T2DM for at least six months and without any known disorder or disability that may prevent them from undertaking regular physical activity..Patients who had advanced stage of diabetic foot ulceration, those who had lower limb amputations, those who were blind and those who were instructed by their physicians to stay away from regular physical activity were excluded. A total of 326 out of the 418 patients receiving treatment in the two hospitals during the data collection period participated in the study. Ethical approval for the study was sought and obtained from the University of Ibadan/University College Hospital Research Ethics Committee (Protocol ID: UI/EC/12/0117).

\section{Procedure for questionnaire translation and data collection}

The Motivation for Physical Activity Questionnaire, the International Physical Activity Questionnaire (IPAQ) as well as the questionnaire to document the socio-demographic characteristics of the participants was translated into Yoruba language using a forward-backward translation process. The translation process was undertaken for the benefit of Yoruba speaking participants who did not understand or speak English. Socio-demographic characteristics comprising age, sex, occupation, level of education and marital status were also documented.

Assessment of motivation for physical activity The Motivation for Physical Activity Questionnaire developed by Deci and Ryan was used to assess the motivation for physical activity among the T2DM patients (Deci and Ryan, 2004; Fitness Management, 2012). The questionnaire is a sixteen-item measure which was designed to classify patients' motivation for physical activity as either intrinsic or extrinsic using factors such as external regulation, introjected regulation, identified regulation, and intrinsic motivation to qualify the type of motivation that spurs the patient into physical activity. An average was taken for each of these factors and these averages were used to calculate the Relative Autonomy Index (RAI) which specifies if the patient is either intrinsically or extrinsically motivated for physical activity. If the value of RAI obtained is negative then, it shows the patient is extrinsically motivated. On the other hand, if the value of RAI calculated is positive then, it means the patient is intrinsically motivated. Intrinsic motivation is defined as the doing of an activity for its inherent 
satisfactions rather than for some separable consequence while extrinsic motivation is a construct that pertains whenever an activity is done in order to attain some separable outcome (Ryan and Deci 2000). The RAI was calculated thus:

The RAI $=$ External regulation $\mathrm{x}(-2)+$ Introjected regulation $\mathrm{x}(-1)+$ Identified regulation $\mathrm{x}(1)+\mathrm{In}$ trinsic motivation x (2) (Deci and Ryan, 2004; Fitness Management, 2012).

Assessment of physical activity: Physical activity was assessed using the International Physical Activity Questionnaire (IPAQ). The questionnaire is aimed at measuring health related physical activity levels in different populations as well as obtaining internationally comparable data on health-related physical activity. There are two forms of the questionnaire, the long and short forms. For this study, the short self-administered form of this questionnaire was used (International Physical Activity Questionnaire, IPAQ, 2012). It is made up of 7 items and 4 domains which cover domestic and yard gardening activity, transport-related activity, leisure time activity and work-related activity. It records the activity in four intensity levels namely vigorous-intensity activity, moderate-intensity activity, walking (low intensity activity) and sedentary. The validity of this instrument has been proven over time by a lot of researches including the 12-country reliability and validity test (Kurtze et al., 2008). It has also been recommended as a cost effective method to assess physical activity (Lee et al., 2011).

\section{Data Analysis}

Descriptive statistics of frequencies and percentages were used to summarize the data and are presented in tables and charts. Logistic regression analyses were carried out on the data of the participants who presented with low physical activity levels. In the analyses, the odds ratio and 95\% confidence intervals for having low physical activity were determined based on motivation type, duration of diagnosis of diabetes and the socio-demographic characteristics. Level of significance was set at $\mathrm{p}<0.05$.

\section{RESULTS}

Socio-demographic characteristics

A total of 326 patients with T2DM participated in this survey. The mean age of the participants was $53.8 \pm 7.4$ years and more than one third of the participants $(38.3 \%)$ were in the age range of 60 to 69 years (Table 1). Female participants (56.1\%) were more than the male participants and most of them were diagnosed within 10 years and below (mean duration of diagnosis $=6.9 \pm 5.7$ years). Table 1 presents other data on level of educational attainment, occupation and marital status of the participants.

Table 1: Descriptive characteristics of the participants

\begin{tabular}{lc}
\hline Variable & $\begin{array}{c}\text { Frequency } \\
(\mathbf{\%})\end{array}$ \\
\hline Age & $26(8.0)$ \\
$<40$ & $81(24.9)$ \\
$40-49$ & $94(28.8)$ \\
$50-59$ & $125(38.3)$ \\
$60-69$ & \\
Sex & $143(43.9)$ \\
Male & $183(56.1)$ \\
Female & \\
Level of education & $54(16.6)$ \\
Primary level & $76(23.3)$ \\
Secondary level & $103(31.6)$ \\
Tertiary level & $42(12.9)$ \\
Postgraduate level & $51(15.6)$ \\
No formal education & \\
Occupation & $177(54.3)$ \\
Gainfully employed & $98(30.1)$ \\
Retiree & $51(15.6)$ \\
Unemployed & \\
Marital status & $11(3.4)$ \\
Single & $249(76.4)$ \\
Married & $66(20.2)$ \\
Widow/widower/separated & \\
Duration of diagnosis (in years) & \\
$\leq 10$ & $207(63.5)$ \\
11-20 & $66(20.2)$ \\
$21-30$ & $38(11.7)$ \\
$31-40$ & $7(2.1)$ \\
$\geq 41$ & $8(2.5)$ \\
\hline
\end{tabular}


Motivation/physical activity in diabetes

Adeniyi et al.,

Motivation and physical activity levels

The type of motivation for physical activity being exhibited by the participants is presented in Figure 1A. The figure shows that most of the participants $(75.2 \%)$ were intrinsically motivated to participate in physical activity programmes, with only $81(24.8 \%)$ of them exhibiting the extrinsic form of motivation. To compute the total scores for the short IPAQ a summation of the duration (in minutes) and frequency (in days) of walking, moderate-intensity and vigorous activities were carried out. The scores are expressed in Metabolic Equivalent (MET)-minutes/ week and are defined as:

walking MET-minutes/week $=3.3 \times$ walking minutes $\mathrm{x}$ walking days;

moderate MET-minutes $/$ week $=4.0 \times$ moderate intensity activity minutes $\mathrm{x}$ moderate days and

vigorous MET-minutes/week $=8.0 \mathrm{x}$ vigorous intensity activity minutes $\mathrm{x}$ vigorous intensity days.

Total physical activity MET minutes/week = sum of walking + moderate + vigorous MET minutes/week scores.

The participants were categorized into three levels: Category 1 (Low activity level) means no activity was reported OR some activity was reported but not enough to meet categories 2 and 3. For Category 2 (Moderate activity level) means a) three or more days of vigorous-intensity activity of at least 20 minutes per day OR b) five or more days of moderate-intensity activity and/or walking of at least 30 minutes per day OR c) five or more days of any combination of walking, moderate-intensity or vigorous-intensity activities achieving a minimum total physical activity of at least 600 MET-minutes/week. In Category 3 (High activity level), it is vigorous-intensity activity on at least three days, achieving a minimum total physical activity of at least 1500 MET-minutes/week OR seven or more days of any combination of walking, moderate-intensity or vigorous-intensity activities achieving a minimum total physical activity of at least 3000 MET-minutes/week (IPAQ, 2012). Figure $1 \mathrm{~B}$ presents the physical activity levels of the participants. About two thirds of the participants (66.9\%) presented with low physical activity levels while only $96(29.4 \%)$ presented with moderate level of physical activity.

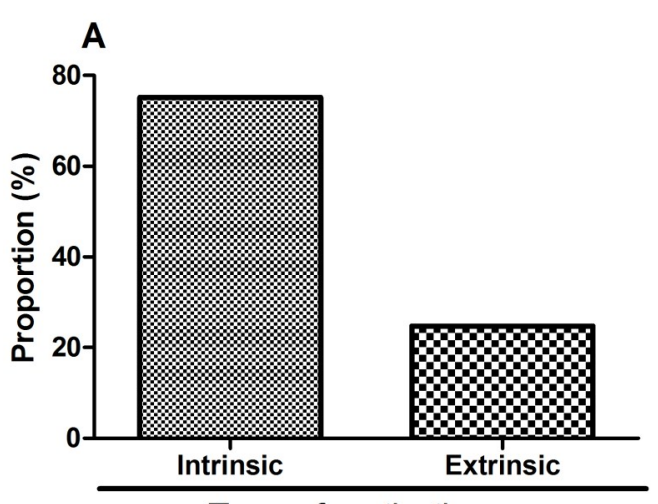

Type of motivation

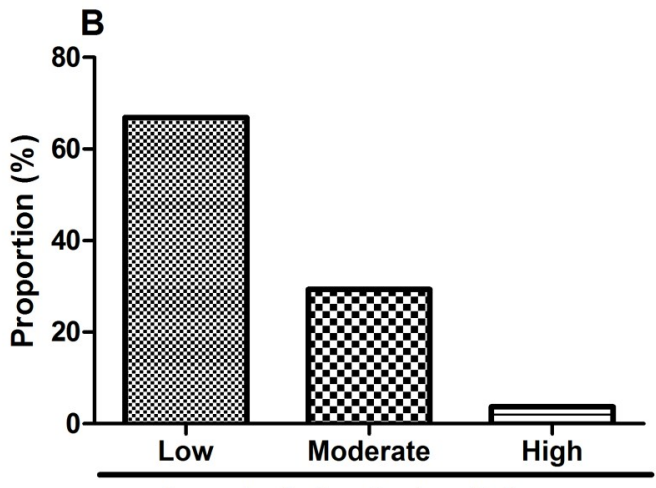

Level of physical activity

Figure 1: Types of motivation exhibited by the participants (A) as well as the levels of physical activity of participants (B).

Association between physical activity and each of motivation and socio-demographic characteristics

As shown in table 2, being extrinsically motivated slightly increased the risk of having low physical activity level but this was not statistically significant $(\mathrm{OR}=1.18 ; 95 \% \mathrm{CI}=0.71-1.36)$ for the group of patients that participated in this study. However, being 60 years and above showed the highest risk of low physical activity when compared with those who were younger than 40 years $(\mathrm{OR}=2.42$; $95 \%$ $\mathrm{CI}=1.91-3.77)$. On the other hand, it was observed that having a tertiary level of education reduced the risk of low physical activity $(\mathrm{OR}=0.52$; $95 \%$ CI $=0.21-0.86)$ while lack of education increased the risk $(\mathrm{OR}=2.21 ; 95 \% \mathrm{CI}=1.64-4.33)$. The duration of diagnosis did not significantly pre- 
dict low level of physical activity, although those who were diagnosed 11 years and over were seen to have higher risk of low physical activity levels.

Table 2: Odds Ratio and $\mathbf{9 5 \%}$ confidence intervals of low physical activity level by motivation and socio-demographic characteristics

\begin{tabular}{ll}
\hline Variable & $\begin{array}{c}\text { Low physical activity } \\
\mathbf{n = 2 1 8} \\
\text { OR (95\% CI) }\end{array}$ \\
\hline $\begin{array}{l}\text { Motivation } \\
\text { Intrinsic }\end{array}$ & 1 \\
Extrinsic & $1.18(0.71,1.36)$ \\
Age (in years) & \\
$<40$ & 1 \\
40-49 & $1.16(0.87,1.98)$ \\
50-59 & $1.55(1.32,1.85)^{*}$ \\
$>$ 60 & $2.42(1.91,3.77)^{*}$ \\
Sex & \\
Male & 1 \\
Female & $1.57(1.22,4.19)^{*}$ \\
Level of education & \\
Primary level & 1 \\
Secondary level & $0.83(0.75,1.44)$ \\
Tertiary level & $0.52(0.21,0.86)^{*}$ \\
No formal education & $2.21(1.64,4.33)^{*}$ \\
Occupation & \\
Gainfully employed & 1 \\
Retiree & $3.89(2.72,6.35)^{*}$ \\
Unemployed & $2.15(1.38,4.28)^{*}$ \\
Marital status & \\
Single & 1 \\
Married & $1.63(1.14,3.66)^{*}$ \\
Widow/widower/ & $1.36(1.11,1,78)^{*}$ \\
separated & \\
Duration of diagnosis (in years) \\
$\leq 10$
\end{tabular}

*Significant at $p<0.05$

\section{DISCUSSION}

This study was conducted to identify the level of physical activity among a group of Nigerian patients with T2DM and to know whether this was influenced by the type of motivational and sociodemographic characteristics that the participants presented with. The findings from this study showed that most of the participants presented with low level of physical activity notwithstanding the fact that most of them were intrinsically motivated towards physical activity. It was also observed that the low level of physical activity presented by this group of patients was not significantly linked with the type of motivation presented by the participants but was significantly linked with the sociodemographic characteristics.

Regular physical activity is generally known to benefit patients with T2DM and this has enjoyed a generous reportage from previously conducted studies. For instance, regular physical activity at moderate to vigorous levels is known to decrease rates of chronic disease and premature mortality (Sigal et al., 2006; Centre for Disease Control and Prevention, CDCP 2008; O'Dougherty et al., 2010). It is however noticed that most of the patients who took part in this study were yet to be physically active at recommended levels in spite of the large amount of evidence to this effect. This observation can be described as the general outlook of events among the general population of which T2DM patients are part of. It has been reported that despite an abundance of evidence surrounding the health benefits associated with physical activity, a large proportion of the adult population continue to lead sedentary and unhealthy lifestyles (O'Dougherty et al., 2010; Caperchione et al., 2012).

The fact that most of the patients presented with intrinsic motivation for physical activity as against extrinsic motivation was an exciting finding because it revealed that the patients were properly motivated to undertake physical activity for the benefit of their overall health. This observation might have originated from the fact that most of them had been diagnosed with T2DM for a few years, a situation that might have given them the opportunity to 
Motivation/physical activity in diabetes

Adeniyi et al.,

develop the right motivation towards physical activity. During this period, the patients may have progressed from the pre-action stage of physical activity to the maintenance stage and specifically, individuals who progressed through these stages would have increased and maintained their levels of selfdetermined motivation (Fortier et al., 2012).

That most of the participants were appropriately motivated to undertake physical activity without a proportionate increase in their physical activity level shows the likelihood of an action disconnect between motivation and the actual performance of the action. This shows that being motivated in itself may not be enough for the T2DM patients to be physically active and it means that some other factors apart from motivation could be responsible. The information-motivation-behavioural skills (IMB) model links performance of health behaviours to the extent to which someone is well informed about the behaviour, motivated to perform the behaviour and has the requisite skills to execute the behaviour (Osborn et al., 2010). Applying the IMB model, the observation from this present study suggests that there is a problem at the motivation-behaviour interphase, with the behaviour in this case being the physical activity. It is not impossible that the sociodemographic characteristics of the participants had served as the moderating factors at this inter-phase.

The IMB model also considers information as vital in determining the behaviour of an individual. However, since the participants in this study demonstrated a high level of motivation, it can be fairly concluded that they were adequately informed because their motivation must have been driven by some information. Hence the need to explore other potential contributing factors. According to Egede and Osborn (2010), it is expected that one who is well informed and motivated to act is likely to develop the skills necessary to drive the behaviour and is likely to benefit maximally. A previous study among patients with diabetes had also reported a disconnection between the information about physical activity and the actual performance of the physical activity. According to a study by Lawton et al., (2006), virtually all the respondents reported awareness about tak- ing regular physical activity as part of diabetes care with most of them describing how they had been encouraged by health professionals to go out for regular walks and sometimes also to swim. However, whilst information about the need to undertake physical activity had clearly been received, only $21.8 \%$ of them claimed to have made a conscious and sustained effort to increase/maintain their physical activity (Lawton et al., 2006). This also implies that some other factors apart from information may be responsible.

Whilst motivation type was not significantly linked, it was observed that the socio-demographic variables (age, sex, occupation, marital status and level of educational attainment) were individually linked significantly with low physical activity among the T2DM patients. The reasons for this observation may not be completely known, but it is possible that the socio-demographic characteristics compared to motivation exert more influence on the low level of physical activity of the T2DM patients making most of them present with low level of physical activity in spite of the appropriate motivation. The sociodemographic variables plausibly have authority over motivation when it comes to physical activity performance and may have contributed to the reasons why a number of studies have reported significant links between physical activity and sociodemographic characteristics. For instance, it was reported that men with T2DM participate in more leisure time physical activity than women do, and those from the highest income group participate in more activity than low- or middle- income groups (Barrett et al., 2007; Huebschmann et al., 2011), while patients with higher education were also reported to be more active (Morreto et al., 2007).

The clinical application of this study lies in the fact that it has helped to show that the issue of motivation, undoubtedly very important among the plethora of reasons why patients with T2DM may not be physically active, may not be the most important barrier to be tackled when it comes to the task of making patients with T2DM from Nigeria to be more physically active. By this, it has helped to broaden the focus of physical activity experts on 
what should be looked out for in patients with T2DM who are not physically active. The study further shows that the physical activity of this group of patients may be improved upon if some other factors other than amotivation are identified and given due considerations. With one major reason for physical inactivity now placed in proper perspectives, it will allow for more focus on how to tackle other likely reasons for physical inactivity among Nigerian patients. From this study and as previously observed by other studies, it appears that socio-demographic characteristics of the T2DM patients will need to be considered seriously if physical activity is to be improved. While it is certain that some of these sociodemographic variables such as age and sex are not modifiable, the idea is that, identifying patients based on these and other potential barriers will provide additional impetus to look closely into strategies of getting patients who fall into these categories to adopt recommended physically activity programmes.

This study has some shortcomings that are worthy of note. The study did not explore the roles of other potential moderators such as the psychosocial confounders. Physical activity of T2DM patients had been observed to be significantly influenced by a combination of psychosocial constructs including the social support, perceived barriers and selfefficacy (Adeniyi et al., 2012). Also, this study did not assess depressive symptoms among the patients and this has been reported to impede self-care behaviours including physical activity (Shahar, 2008; Egede and Osborn, 2010). Because the study sample was hospital based, it is recommended that caution should be exercised in generalizing the findings as the participants in this study may not accurately represent the entire population of patients with diabetes who may not have presented in the hospitals. This is because unlike the non-hospital patients, the patients visiting the hospitals may have had access to a variety of health education on self-care efforts which may have influenced their motivation and physical activity levels. These weaknesses notwithstanding, the strength of this study lies in the fact it has shown the need to consider other factors in order to physi- cally work up patients with T2DM even when they present with the appropriate motivations.

\section{CONCLUSIONS AND RECOMMENDA- TIONS}

The Nigerian patients with T2DM are appropriately motivated to do physical activity but their physical activity is largely below recommended levels. Although motivation is very important when it comes to physical activity participation, this study has brought to the fore that there is also the need to look into other issues outside motivation, such as the socio-demographic characteristics of the patients with T2DM in order to achieve an increase in their physical activity to the recommended levels. Since physical activity level and all variables that influence participation are never static, the challenge is for the healthcare workers in this field to identify the dynamics of physical activity and inactivity as relates to many other aspects of life of a patient with T2DM. We recommend longitudinal studies that will look into the dynamism of the multiple life events of patients with T2DM in order to identify those that improve or reduce their physical activity levels.

\section{ACKNOWLEDGEMENT}

Our special thanks to the T2DM patients of the University College Hospital and Ring Road State Hospital, Ibadan, Nigeria for their kind patience in filling our questionnaires.

\section{COMPETING INTERESTS}

The authors declare that they have no competing interests.

\section{REFERENCES}

Adeniyi, A.F., Idowu, O.A., Ogwumike, O.O., and Adeniyi, C.Y. (2012). Comparative influence of self-efficacy, social support and perceived barriers on low physical activity development in patients with type 2 diabetes, hypertension or stroke. Ethiopian Journal of Health Sciences, 22, 113-119.

Amico, K.R. (2011). A situated-Information Motivation Behavioral Skills Model of care initiation and maintenance (sIMB-CIM): an 
Motivation/physical activity in diabetes

Adeniyi et al.,

IMB Model based approach to understanding and intervening in engagement in care for chronic medical conditions. Journal of Health Psychology, 16, 1071-1081.

Barrett, J.E., Plotnikoff, R.C., Courneya, K.S., and Raine, K.D. (2007). Physical activity and type 2 diabetes: exploring the role of gender and income. The Diabetes Educator, 33, 128 $-143$.

Biddle, S.J.H., and Nigg, C.R. (2000). Theories of exercise behavior. International Journal of Sport Psychology, 31, 290-304.

Caperchione, C.M., Vandelanotte, C., Kolt, G.S., Duncan, M., Ellison, M., George, E. et al. (2012). What a man wants: understanding the challenges and motivations to physical activity participation and healthy eating in middle-aged Australian men. American Journal of Mens Health, 6, 453-461.

Center for Disease Control and Prevention (2008). 2008 Physical Activity Guidelines for Americans Fact Sheet for Health Professionals on Physical Activity Guidelines for Adults. http://www.cdc.gov/nccdphp/dnpa/ physical/pdf/PA Fact Sheet Adults.pdf Retrieved 14 March, 2013, 6:45 pm.

Deci, E.L., and Ryan, R.M. (2004). Exercise SelfRegulation Questionnaires. SelfDetermination Theory: An Approach to Human Motivation and Personality-The Self-Regulation Questionnaires. http:// selfdeterminationtheory.org/questionna ires/10- questionnaires/48; Accessed 14 December, 2012; 4:40 pm.

Dunbar-Jacobe, J., and Mortimer-Stephens, M.K. (2001). Treatment adherence in chronic disease. Journal of Clinical Epidemiology, 54, S57-S60.

Egede, L.E. and Osborn, C.Y. (2010). Role of motivation in the relationship between depression, self-care, and glycemic control in adults with type 2 diabetes. The Diabetes Educator, 36, 276-283.

Fitness Management (2012) Module B: specific leaning outcomes. www.edu.gov.mb.ca/k12/ cur/physhlth/frame found.../4 fm.pdf; Accessed 6 March, 2012; 7:30 pm
Fortier, M.S., Sweet, S.N., Tulloch, H., Blanchard, C.M, Sigal, R.J., Kenny, G.P. et al (2012). Self-determination and exercise stages of change: results from the Diabetes Aerobic and Resistance Exercise Trial. Journal of Health Psychology, 17, 87-99.

Huebschmann, A.G., Crane, L.A., Belansky, E.S., Scarbro, S., Marshall, J.A., and Regensteiner, J. G. (2011). Fear of injury with physical activity is greater in adults with diabetes than in adults without diabetes. Diabetes Care, 34, 1717-1722.

International Physical Activity Questionnaire (2012) www.ipaq.ki.se/contact/html; Retrieved at 6:34 pm on 21/2/2012.

Jeon, C.Y., Lokken, R.P., Hu, F.B., and van Dam, R. M. (2007). Physical activity of moderate intensity and risk of type 2 diabetes. a systematic review. Diabetes Care, 30, 744 752.

Korkiakangas, E.E., Alahuhta, M.A., and Laitinen, J.H. (2009). Barriers to regular exercise among adults at high risk or diagnosed with type 2 diabetes: a systematic review. Health Promotion International, 24, 416427.

Kurtze, N., Rangul, V., and Hustvedt, B. (2008). Reliability and validity of the international physical activity questionnaire in the NordTrøndelag health study (HUNT) population of men. BMC Medical Research Methodology, 8, 63 doi:10.1186/1471-2288-8-63.

Lawton, J., Ahmad, N., Hanna, L., Douglas, M., and Hallowell, N. (2006). 'I can't do any serious exercise': barriers to physical activity amongst people of Pakistani and Indian origin with Type 2 diabetes. Health Education Research, 21, 43-54.

Lee, P.H., Macfarlane, D.J., Lam, T.H., and Stewart, S.M. (2011). Validity of the international physical activity questionnaire short form (IPAQ-SF): A systematic review. International Journal of Behavioral Nutrition and Physical Activity, 8, 115 doi:10.1186/1479-5868-8115.

Mori, D.L., Silberbogen, A.K., Collins, A.E., Ulloa, E.W., Brown, K.L. and Niles, B.L. (2011). 
Promoting physical activity in individuals with diabetes: telehealth approaches. Diabetes Spectrum, 24, 127-135.

Morrato, E.H., Hill, J.O., Wyatt, H.R., Ghushchyan, V., and Sullivan, P.W. (2007). Physical activity in U.S. adults with diabetes and at risk for developing diabetes. Diabetes Care, 30, 203 209.

O'Dougherty, M., Kurzer, M.S., and Schmitz, K.H. (2010). Shifting motivations: young women's reflections on physical activity over time and across contexts. Health Education and Behaviour, 37, 547-567.

Osborn, C.Y., Amico, K.R., Fisher, W.A., Egede, L.E. and Fisher, J.D. (2010). An Information Motivation-Behavioral skills analysis of diet and exercise behavior in Puerto Ricans with diabetes. Journal of Health Psychology, 15, 1201-1213.

Plotnikoff, R.C., Brez, S., and Hotz, S.B. (2010). Exercise behavior in a community sample with diabetes: understanding the determinants of exercise behavioral change. The Diabetes
Educator, 26, 450-459.

Ryan, R.M., and Deci, E.L. (2000). Intrinsic and extrinsic motivations: classic definitions and new directions. Contemporary Educational Psychology, 25, 54-67.

Shahar, J. (2008). Helping your patients become active. Diabetes Spectrum, 21, 59-62.

Shigaki, C., Kruse, R.L., Mebr, D., Sheldon, K.M., Ge, B., Moore, C. et al. (2010). Motivation and diabetes self-management. Chronic Illness, 6, 202-214.

Sigal, R.J., Kenny, G.P., Wasserman, D.H., Castaneda-Sceppa, C., and White, R.D. (2006). Physical Activity/Exercise and Type 2 Diabetes: A consensus statement from the American Diabetes Association. Diabetes Care, 29, 1433-1438.

Trost, S.G., Pate, R.R., Sallis, J.F., Freedson, P.S., Taylor, W.C., Dowda, M. et al (2002). Age and gender differences in objectively measured physical activity in youth. Medicine and Science in Sports and Exercise, 34, 350 -355 .
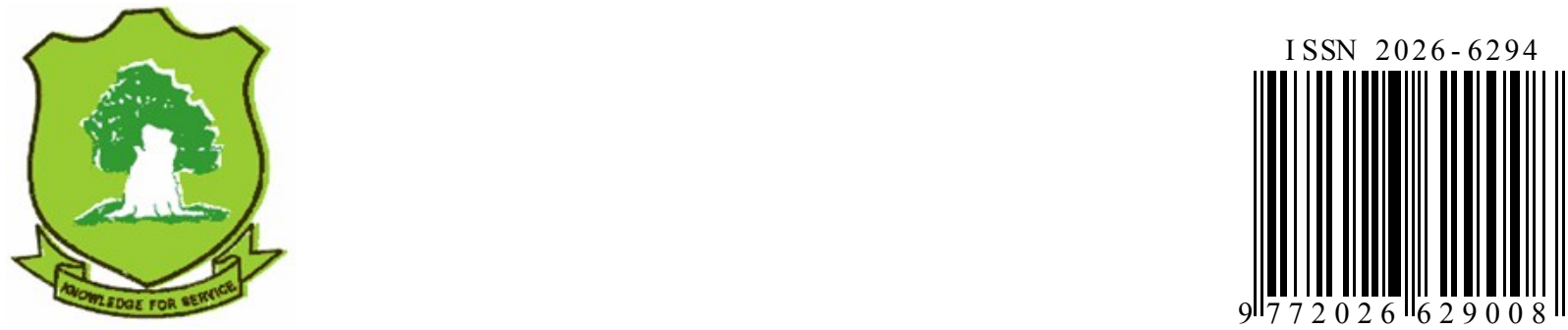